\title{
Anterior rectus sheath flap for closure of the abdominal wall defects
}

\author{
Alaa A El-Sisy, $M D$ \\ Department of General Surgery, Menoufiya University, Egypt.
}

\begin{abstract}
Introduction: The most important functions of the abdominal wall are protection and compression. Reconstruction of large abdominal wall defects following trauma, abdominal wall tumor resection and recurrent hernia represents a great challenge. Multiple techniques have been described to restore the integrity of the abdominal wall as primary fascial repair, component separation, and interpositional materials.

Aim of the work: To evaluate the effectiveness of the anterior rectus sheath flap to bridge the fascial defect with supporting polypropylene mesh.

Patients \& methods: Twenty nine patients with abdominal wall defects were managed at Menoufiya University Hospital between January 2005 and October 2009 using the anterior rectus sheath flap with supporting polypropylene mesh.

Conclusion: This technique will be added as one of the methods of closing abdominal wall defects that can be used in appropriate patients. The clear indication, advantages, disadvantages, and long term outcomes compared with other methods will be hopefully explored by more time and studies.
\end{abstract}

Keywords: Abdominal defects, rectus sheath flap, supporting mesh.

\section{Introduction:}

The most important functions of the abdominal wall are protection and compression. Reconstruction of large abdominal wall defects following trauma, abdominal wall tumor resection and recurrent hernia represents a great challenge. ${ }^{1}$

Large abdominal wall defects may have both cutaneous and fascial defects. ${ }^{2}$

Multiple techniques have been described to restore the integrity of the abdominal wall, although the indications and applications can be difficult to navigate. ${ }^{3}$

Primary fascial repair, component separation, and interpositional materials such as biological or synthetic mesh or tensor fascia latae grafts are associated with many complications as hernia recurrence, wound infection, enterocutaneous fistulas and bowel adhesion. ${ }^{4}$

It has been well documented that primary fascial repair alone has a high incidence of recurrence. ${ }^{5}$
If there is insufficient fascia, there are two options: Component separation or interpositional mesh. Component separation involves incision of the external oblique fascia and muscle lateral to the rectus abdominis muscle, resulting in medial advancement of the rectus abdominis muscles. This facilitates primary closure of fascia with less tension. ${ }^{4}$

Mesh is used as an interpositional material to bridge the fascial defect when primary repair is not possible and is sutured underneath and several centimeters beyond the leading fascial edge. ${ }^{4}$

There are three major considerations when selecting a specific synthetic product: Contact with the bowel, incorporation into the surrounding tissue and risk of wound breakdown leading to mesh exposure. Polypropylene evokes a vigorous inflammatory response that can lead to dense adhesions and increased risk for fistula formation. This inflammatory reaction is less marked when polytetrafluoroethylene has been used. So, 
when fascial closure is possible, it is preferred and mesh is used as an onlay mesh. 6,7

A cellular dermal (AlloDerm) matrix may be used as biological material to bridge the fascial defect, not as onlay mesh. The cellular dermal matrix has three distinct advantages over synthetic material. First, there is revascularization, which reduces the risk of infection. Second, there is minimal inflammatory reaction and thus fewer bowel adhesion. Third, when wound breakdown occurs, there is no need for removal of the cellular dermal matrix. ${ }^{8}$

However, AlloDerm is expensive and the cost must be justified. ${ }^{9}$

Tensor fascia latae is another biological option; however, this is less dynamic and thus is associated with a higher incidence of recurrence. ${ }^{10}$

\section{Aim of the work:}

The aim of this work is to evaluate the effectiveness of the anterior rectus sheath flap to bridge the fascial defect with supporting polypropylene mesh.

\section{Patients and methods:}

Twenty nine patients with abdominal wall defects, either following abdominal wall tumor resection or large hernial defect, were managed at Menoufiya University Hospital between January 2005 and October 2009 using the anterior rectus sheath flap with supporting polypropylene mesh. Abdominal wall defects followed desmoid tumor resection in 10 cases $(50 \%)$, soft tissue sarcoma in 3 cases $(15 \%)$, incisional hernia in 9 cases $(45 \%)$ and recurrent hernia in 7 cases $(35 \%)$.

\section{Techniques:}

The abdominal wall defect followed tumor resection of the abdominal wall either in Desmoids tumor or soft tissue sarcoma or following excision of the sac after reduction of the content. The abdominal wall defects were assessed as regarding their size in two dimentions; the length and width in $\mathrm{cm}$. The site in the abdominal wall (Central defect, unilateral defect, above or below the umbilicus or affecting above and below), and the condition of the abdominal wall muscles and rectus sheath were assessed.

The procedure is started by separating the skin and subcutaneous tissue from the anterior rectus sheath to create skin flap which will be used later on to correct skin defect if present.

The anterior rectus sheath flap is fashioned by longitudinal incision along the entire length of the lateral border of the rectus sheath. This incision may be done unilateral or bilateral according to the site of the defect.

The site of this incision must be chosen carefully to avoid entering at the conjoined tendon of the internal oblique apponeurosis and the external oblique aponeurosis. The anterior sheath was then dissected from lateral to medial, freeing it from the rectus muscle until the meating between the anterior and posterior sheath.

Polypropylene 0 suture was used in continuous manner to reinforce the junction between the anterior and posterior sheath. Then the anterior sheath flap was used to bridge the defect and was sutured by polypropylene 0 to the other edge of the defect. After fascial closure, supporting polypropylene mesh was used to cover the exposed rectus muscle and the new fascial closure and extending about $2 \mathrm{~cm}$ lateral to the edge and fixed by polypropylene 0 suture at 2 points: The first at the edge of the defect and the lateral remaining part of sheath over the rectus muscle. The second line of fixation is about $2 \mathrm{~cm}$ lateral from the first fixation. Then closure of the skin and subcutaneous tissue with suction drainage is done.

\section{Post-operative management:}

Broad spectrum I.V. antibiotic was used for 3 days followed by broad spectrum oral antibiotic for 5 days with good analgesic. Abdominal belt was used to decrease postoperative seroma. The drain was removed when it revealed less than $50 \mathrm{cc}$ in 2 successive days. 


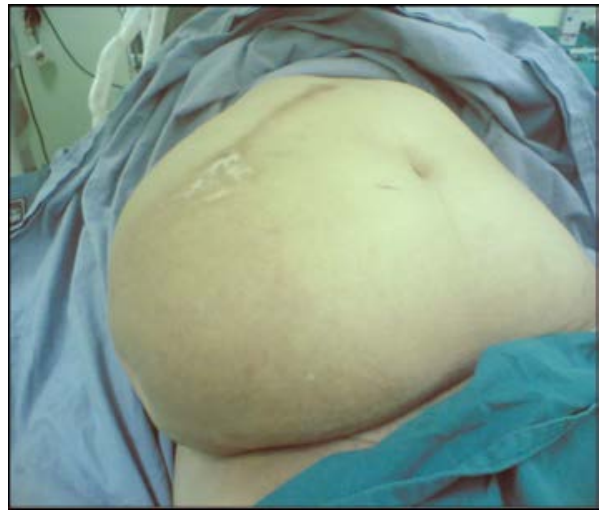

Figure (1): Huge hernia.

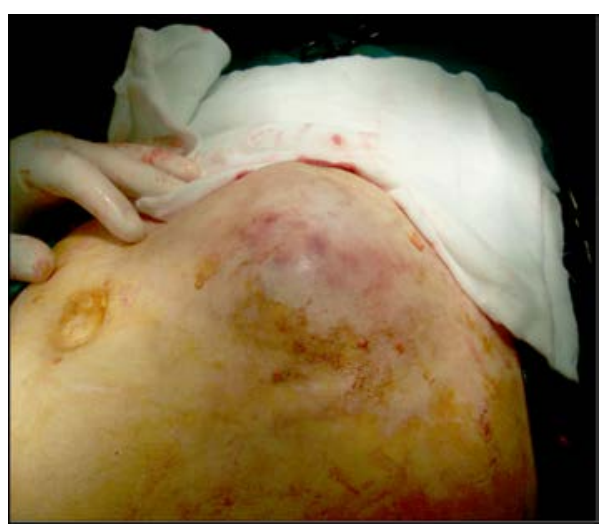

(A)

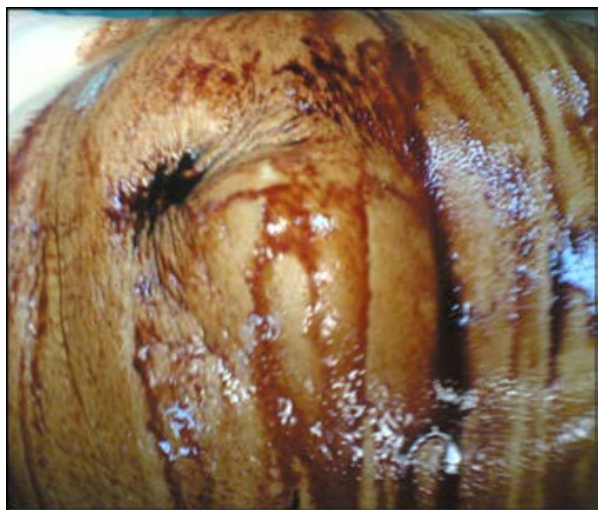

Figure (2): Abdominal wall soft tissue sarcoma.

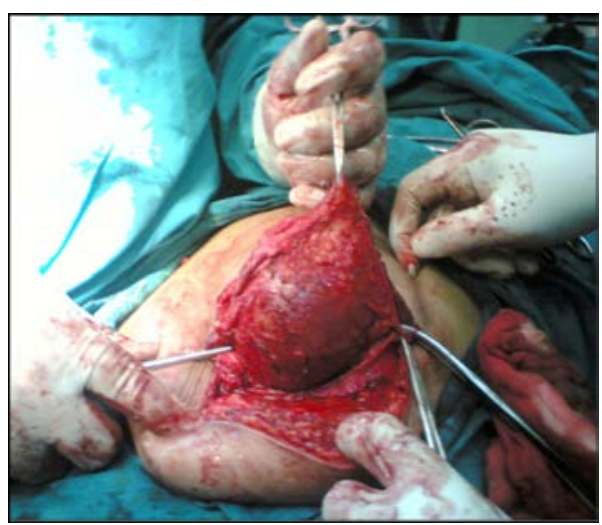

(B)

Figure (3): Excision of desmoid tumor.

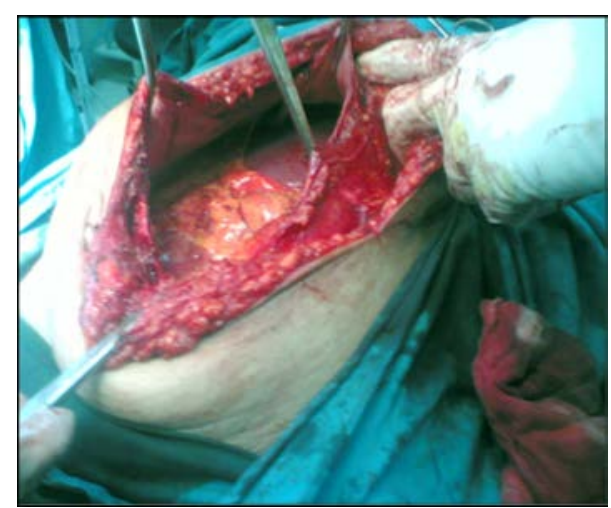

(A)

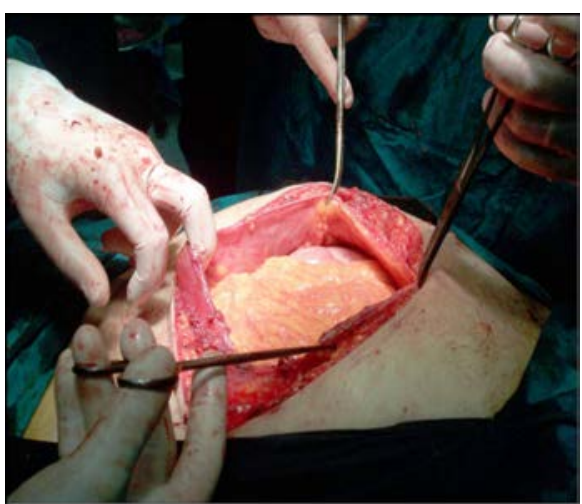

(B)

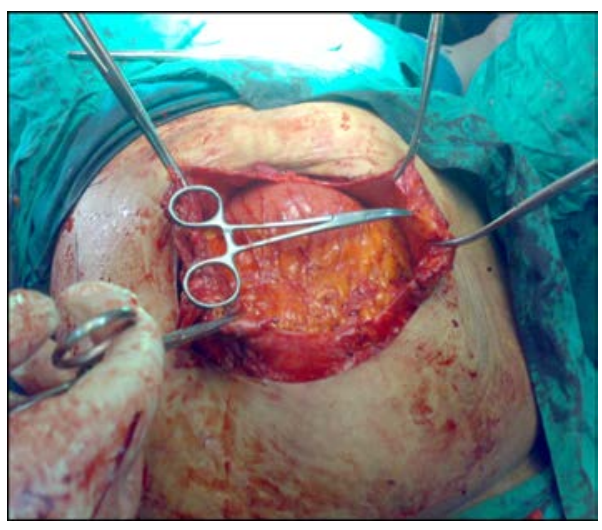

(C)

Figure (4): Abdominal defect. 


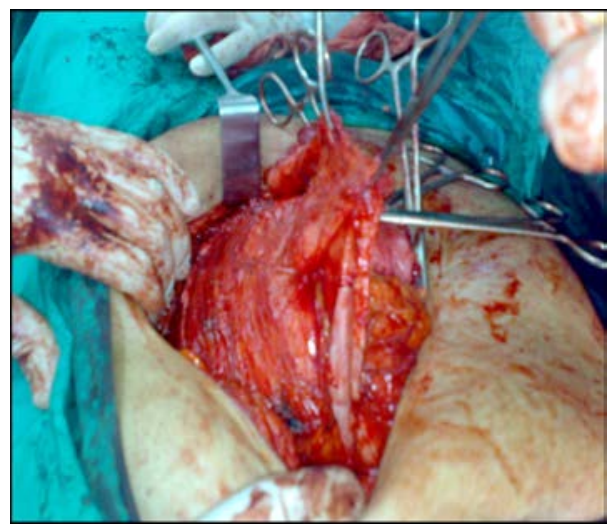

Figure (5): Elevation of whole anterior rectus sheath flap.

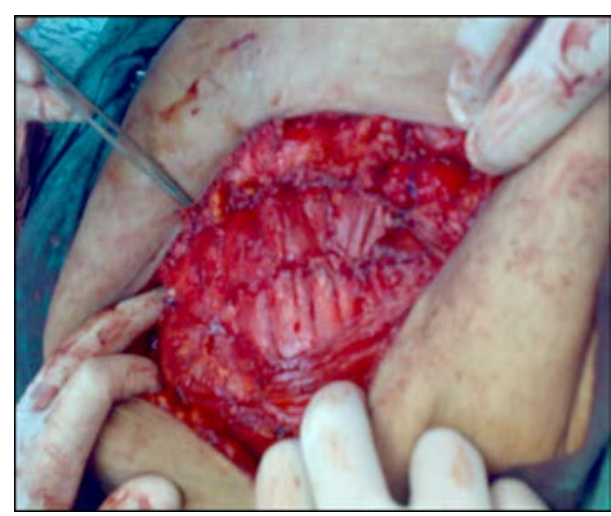

Figure (7): Covering the defect using the flap.

\section{Results:}

This study was carried out on 29 patients, 16 females (55.2\%) and 13 males (44.8\%), with an average age of 36 years (range 19-67 years). Ten cases $(34.5 \%)$ are of desmoids

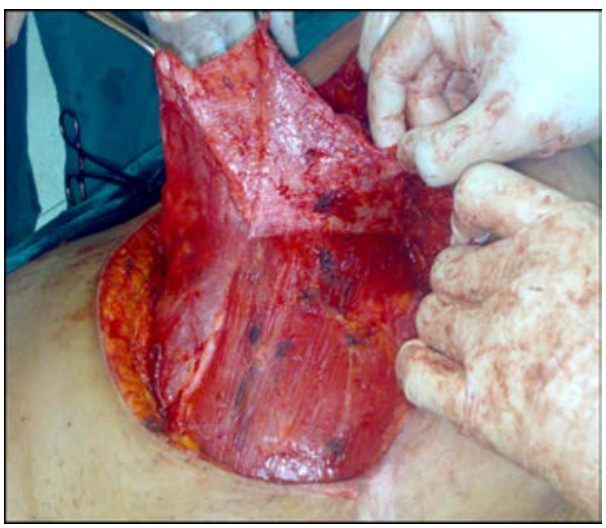

Figure (6): Elevation of part of anterior rectus.

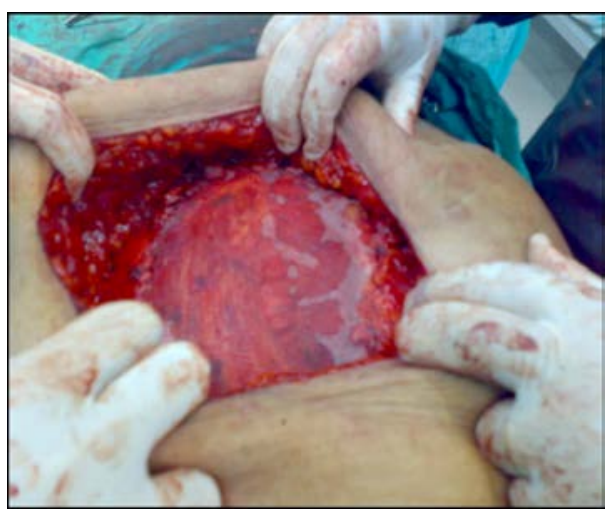

Figure (8): Using supporting mesh to cover the flap and rectus muscle.

tumor resection, 9 cases $(31.1 \%)$ of incisional hernia, 7 cases $(24.1 \%)$ of recurrent abdominal wall hernia and 3 cases $(10.3 \%)$ are after soft tissue sarcoma resection.

Table (1): Demographic and peri-operative data.

\begin{tabular}{|l|c|}
\hline$\bullet$ Male / female & $16 / 13$ \\
\hline$\bullet$ Age in years & 36 years $(19-67)$ \\
\hline$\bullet$ Body mass index $\left(\mathrm{kg} / \mathrm{m}^{2}\right)$ & $33(18-51)$ \\
\hline$\bullet$ Defect size $\left(\mathrm{cm}^{2}\right)$ & $160(100-240)$ \\
\hline$\bullet$ Mesh size & $450(300-600)$ \\
\hline$\bullet$ Mesh defect ratio & 2.8 \\
\hline$\bullet$ Operating time $($ minutes $)$ & $140(70-240)$ \\
\hline$\bullet$ Estimate blood loss $(\mathrm{ml})$ & $190(100-750)$ \\
\hline$\bullet$ Postoperative stay & 6 days (3-9) \\
\hline
\end{tabular}


Table (2): Co-morbidities, data are expressed as absolute value.

\begin{tabular}{|l|c|c|}
\hline$\bullet$ None & 16 & $55.2 \%$ \\
\hline$\bullet$ Obesity (BMI) & 4 & $13.8 \%$ \\
\hline$\bullet$ Diabetes & 7 & $24.1 \%$ \\
\hline$\bullet$ Hypertension & 10 & $34.5 \%$ \\
\hline$\bullet$ Heavy smoking & 9 & $31 \%$ \\
\hline$\bullet$ Hepatic disease & 6 & $20.7 \%$ \\
\hline$\bullet$ Ischemic heart disease & 5 & $17.2 \%$ \\
\hline
\end{tabular}

Table (3): Postoperative complications.

\begin{tabular}{|l|c|c|}
\hline Early postoperative complications & & \\
- Wound infection & 4 & $13.8 \%$ \\
- Haematoma & 1 & $3.4 \%$ \\
- Seroma & 6 & $20.7 \%$ \\
\hline Late postoperative & & \\
- No recurrence, no intestinal fistula, & 0 & $0 \%$ \\
no intestinal obstruction and no mesh removal & & \\
- Pouching out & 2 & $6.9 \%$ \\
\hline
\end{tabular}

\section{Discussion:}

Many techniques have been developed to re-establish the integrity of the abdominal wall, including primary fascial closure, component separation and interpositional materials such as biological or synthetic mesh or tensor fascia latae grafts. ${ }^{4}$

The primary fascial closure is the simplest option but the recurrence rate is reported to be as high as $54 \%$ and so is often unwise. ${ }^{11}$

When there is difficulty in advancing the fascial edge toward the midline, component separation provides additional length, thus permits movement of the edge toward midline. ${ }^{9}$

The techniques of component separation obviates the need for synthetic material to be in contact with bowel and thus reduces the risk of enterocutaneous fistula and intestinal adhesion. ${ }^{9}$

Also, in our study, the synthetic material will not be indirect contact with bowel and no enterocutaneous fistula or intestinal obstruction was reported in postoperative follow up. Also,
Kushimoto et al. found that early fascial closure using bilateral rectus sheath flap for management of acute open abdomen was not associated with fistula and intestinal obstruction. ${ }^{5}$

According to Ramirez ${ }^{4}$ study in component separation, the total advancement to the midline are $5,10,3 \mathrm{~cm}$ on either side in the epigastric, umbilical and suprapubic regions respectively. So, the benefit of this technique is for central defects only and using both sides and has no value if there is lateral defect, when unilateral rectus sheath and muscle was excised as in abdominal wall tumor (Desmoid tumor).

Also, the component separation needs more dissection, and more length time, and produces more weakness in the lateral abdominal wall as it produces gap in external oblique which is the strongest part of the abdominal wall. As regards the anterior rectus sheath flap technique, it can gap large central or lateral abdominal wall defect (width $10 \mathrm{~cm}$, length $20 \mathrm{~cm}$ ) and can be done in short time with no need for 
more dissection when comparing the anterior with other technique. For gaping abdominal defect, it is a simple technique that can be done by all surgeons and is of low cost when comparing it with using cellular dermal matrix when used for covering the abdominal wall defect.

Although of short term result, it may be expected that a single layered abdominal wall will give in with time (out pouching) with increased abdominal pressure. This problem may be even greater below the arcuate line, where the posterior rectus sheath is entirely absent. So, we use a supporting (polypropylene mesh) to reduce the incidence of this outpouching. It is often difficult to distinguish between outpouching and a true recurrent hernia.

\section{Conclusion:}

We conclude that this technique will be added as one of the methods of closing abdominal wall defects that can be used in appropriate patients. The clear indication, advantages, disadvantages, and long term outcomes compared with other methods will be hopefully explored by more time and studies.

\section{References:}

1- Eduardo DR, Rachel BL, Ronald PS, et al: Abdominal wall reconstruction following sever loss of domain: The R Adams Cowley Shock Trauma Center Algorithm. Plast Reconstr Surg 2007; 120: 669.

2- Jernigan TW, Fabian TG, Groee MA, et al: Staged management of gaint abdominal wall defects. Ann Surg 2003; 238: 349.

3- Fabian, TG; Groee, MA; Pritchard, F, et al: Planned ventral hernia: staged management for acute abdominal defects. Ann Surg 1994; 219: 643.
4- Ramierz OM, Ruas E, Dellon AL: "Components Separation" method for closure of abdominal wall defects: an anatomic and clinical study. Plast Reconstr Surg 1990; 86: 519-526.

5- Kushimoto S, Yamamoto Y, Aiboshi J, et al: Usefulness of the bilateral anterior rectus abdominis sheath turnover flap method for early fascial closure in patients requiring open abdominal management. World J Surg 2008; 31: 2-8.

6- Butler GF, Prieto VG: Reduction of adhesions with composite AlloDerm/polypropylene mesh implants for abdominal wall reconstruction. Plast Reconstr Surg 2004; 114: 464.

7- DeSagun EZ, Botts JL, Srivastava A, et al: Long term outcome of xenogenic dermal matrix implantation in immunocompotent rats. J Surg Res 2001; 96: 96.

8- Eppley B: Experimental assessment of the revascularization of a cellular human dermis for soft-tissue augmentation. Plast Reconstr Surg 2001; 107: 757.

9- Giratto JA, Chiaramonte M, Menon N, et al: Recalcitrant abdominal wall hernias: long-term superiority of autologous repair. Plast Reconstr Surg 2003; 112: 106. 10-Sukkar SM, Dumanian GA, Szezerba SM, et al: Challenging abdominal wall defects. Ann J Surg 2002; 181: 115.

11-Robinson TN, Clarke JH, Walsh MD: Major mesh-related complications following hernia repair; events reported to the food and drug administration. Surg Endose 2005; 19: 1556. 